\title{
Produção científica sobre a COVID-19 no Brasil: uma revisão de escopo
}

\section{Scientific production on COVID-19 in Brazil: a scoping review}

\author{
Daniel Marques Motal,* \\ Paulo José Gonçalves \\ Ferreira' (iD
}

Lisiane Freitas Leal"
' Agência Nacional de Vigilância Sanitária (Anvisa), Brasília, DF, Brasil

" McGill University, Montreal, QC, Canadá

\section{* E-mail: dmarques2003@yahoo.com.br}

Recebido: 11 maio 2020 Aprovado: 18 maio 2020

\section{RESUMO}

Introdução: A produção científica nacional sobre a COVID-19 tem papel imediato na formulação de políticas de enfrentamento da doença e na orientação de decisões clínicas. Objetivo: Identificar e caracterizar a produção científica sobre assuntos relacionados à COVID-19 no Brasil em revistas nacionais a partir de artigos publicados entre $1^{\circ}$ de dezembro de 2019 e 2 de maio de 2020. Método: Revisão de escopo, cuja busca por artigos ocorreu na Coleção SciELO Brasil e nos sítios eletrônicos das revistas Visa em Debate e Ciência \& Saúde Coletiva. O banco de dados validado foi submetido a uma análise quantitativa simples para fornecer resumos numéricos das características de interesse da literatura incluída na revisão. Resultados: Foram incluídos 58 (20,8\%) artigos oriundos de 22 revistas nacionais. 0 maior número de artigos proveio das revistas que desenvolveram opções rápidas de publicação ou vinham adotando um modelo de publicação de fluxo contínuo ( $n=45,77,6 \%$ ). Os artigos foram enquadrados em quatro categorias, dentre as sete definidas: comentário $(n=43,74,1 \%)$, estudo descritivo $(n=8$, $13,8 \%)$, revisão da literatura $(n=6,10,4 \%)$ e estudo analítico $(n=1,1,7 \%)$. Constatou-se apenas uma revisão sistemática e o estudo analítico publicado foi do tipo ecológico. 0 mês de abril concentrou $86,2 \%$ dos artigos, e o seu maior pico, compreendendo o período estudado, ocorreu em 9 de abril, com oito publicações. Dos 58 artigos, o "Isolamento social, saúde mental e outros aspectos relacionados a comportamentos sociais" foi o tema mais prevalente $(n=14,24,1 \%)$. Conclusões: Esta revisão de escopo produziu um mapa da produção científica sobre a COVID-19 no Brasil. Há lacunas importantes, principalmente no que tange aos ensaios clínicos randomizados e estudos de coorte, que precisam ser preenchidas com a realização de pesquisas.

PALAVRAS-CHAVE: Artigo de Revista; Infecções por Coronavírus; COVID-19; Pandemias; Revisão de Escopo

\section{ABSTRACT}

Introduction: The national scientific production on COVID-19 has an immediate role in developing policies to tackle the disease and in guiding clinical decisions. Objective: To identify and characterize the scientific production on topics related to COVID-19 in Brazil in national journals from articles published between December 1, 2019, and May 2, 2020. Method: Scoping review, whose search for articles occurred in the SciELO Collection Brazil and on the websites of journal Visa em Debate and Ciência \& Saúde Coletiva. The validated database was assessed by a simple quantitative analysis to provide numerical summaries of the characteristics of interest in the literature included in the review. Results: 58 (20.8\%) articles from 22 national journals were included. The largest number of articles came from journals that developed fast publishing options or that had been adopting a continuous flow publication model $(n=45,77.6 \%)$. The articles were framed in four categories, among seven defined: Comment $(n=43,74.1 \%)$, Descriptive study $(n=8,13.8 \%)$, Literature review $(n=6,10.4 \%)$ and Analytical study $(n=1,1.7 \%)$. Only one systematic review was found and the analytical study was classified as an ecological study. April concentrated $86.2 \%$ of the articles published, with the peak of publications 
occurring on April 9 (8 articles). Among 58 articles, "Social isolation, mental health and other aspects related to social behaviours" was the most prevalent theme $(n=14,24.1 \%)$. Conclusions: This scoping review produced a map of scientific production on COVID-19 in Brazil. There are important gaps, especially concerning randomized clinical trials and cohort studies, which need to be filled on further research in our country.

KEYWORDS: Journal Article; Coronavirus Infections; COVID-19; Pandemics; Scoping Review

\section{INTRODUÇÃO}

A doença COVID-19 é causada pelo coronavírus da Síndrome Respiratória Aguda Grave 2 (SARS-CoV-2). Este vírus foi identificado pela primeira vez em um grupo de pacientes com pneumonia atípica em Wuhan, China, em $1^{\circ}$ de dezembro de $2019^{1}$. Em 30 de janeiro, a Organização Mundial da Saúde declarou a COVID-19 uma emergência de saúde pública de interesse internacional e, logo em seguida, uma pandemia, em 11 de março de $2020^{2}$. Transcorridos 167 dias, mais de 4,7 milhões de casos foram confirmados com 314.476 óbitos (17/5/2020, 18 h $32 \mathrm{~min}$ $18 \mathrm{seg}$ ) em 188 países/regiões ${ }^{3}$.

No Brasil, o primeiro caso foi confirmado em 26 de fevereiro de $2020^{4}$, enquanto o primeiro óbito ocorreu 20 dias depois, em 17 de março. Até o momento (17/05/2020, 18 h $32 \mathrm{~min} 18 \mathrm{seg})$, foram registrados 236.131 casos diagnosticados e 15.776 óbitos distribuídos nas cinco regiões do país ${ }^{3}$. Neste momento, há, ainda, muitas dúvidas sobre o manejo da doença e suas implicações para a população, o sistema de saúde e a economia.

Nesse tipo de emergência em saúde pública de rápida disseminação geográfica, elevada gravidade e com muitas lacunas de conhecimentos, é esperado que se intensifiquem as buscas por informações na literatura científica, bem como ocorra aumento na publicação de artigos científicos sobre o tema ${ }^{5}$. A disponibilização pública, preferencialmente no idioma do país, de artigos científicos revisados por pares, ao longo do curso da pandemia, é essencial para apoiar o conjunto de decisões clínicas e de gestão pública e de vigilância em saúde, incluindo a vigilância sanitária.

Entretanto, o enfrentamento da COVID-19 pautado nessa premissa não é uma estratégia simples em países, como o Brasil. Diferenças regionais na densidade demográfica, urbanização, estrutura de saúde e aspectos sociais e econômicos ${ }^{4}$, bem como diversos tipos de desigualdades, a exemplo, da dificuldade no acesso à informação científica de qualidade ${ }^{6}$, são reais e preocupantes.

Nesse contexto, especificamente no Brasil, pode ser um grande desafio para os gestores, médicos, demais profissionais da saúde e população em geral, efetivamente se apropriarem de evidências científicas publicadas em periódicos internacionais, em sua maioria no idioma em inglês, sobre diversos aspectos relacionados à COVID-19. O Brasil é o único país da América Latina cujo idioma oficial é exclusivamente o Português, e a população apresenta baixíssima proficiência na língua Inglesa. Segundo levantamento do British Council, apenas 5,0\% dos brasileiros afirmam ter conhecimento de inglês, variando de 3,5\% entre adultos de 35 e 50 anos a 10,3\% entre jovens de 18 e 24 anos de idade. Dessa população, 16,0\% têm nível avançado/fluente contra 47,0\% com nível básico de inglês ${ }^{7}$.

Considerando-se o massivo aumento do número de publicações em um idioma e a necessidade de compreender a produção científica sobre um determinado assunto em tempos de pandemia, as revisões de escopo são consideradas ferramentas válidas para mapear evidências disponíveis, descrever as características do corpo da literatura, esclarecer conceitos-chave e identificar lacunas de conhecimento ${ }^{8}$.

Em meio à urgência de mapear evidências de qualidade sobre a COVID-19 disponíveis, principalmente em idioma português, como forma de facilitar uma apropriação mais efetiva para quem delas necessita, bem como identificar as lacunas de conhecimentos necessários ao contexto do país, os autores entenderam que a realização de uma revisão do escopo é considerada adequada para contribuir com tal situação.

Assim, o objetivo desta revisão de escopo foi identificar e caracterizar a produção científica sobre assuntos relacionados à COVID-19 no Brasil em revistas nacionais a partir de artigos publicados entre $1^{\circ}$ de dezembro de 2019 e 2 de maio de 2020 (10 h $02 \mathrm{~min}$ ).

\section{MÉTODO}

\section{Desenho do estudo}

Esta revisão de escopo seguiu recomendações propostas pelo Preferred Reporting Items for Systematic reviews and Meta-Analyses extension for Scoping Reviews (PRISMA-SCR) ${ }^{9}$ e contidas no Joanna Briggs Institute Reviewer's Manual ${ }^{10}$. A revisão envolveu a formulação de uma pergunta de pesquisa para orientar o estudo: "Como se caracteriza a produção científica sobre a COVID-19 em revistas nacionais?”. A revisão foi realizada entre os dias 25 de abril e 8 de maio de 2020 .

\section{Estratégias de buscas}

Como estratégias de buscas, foram realizadas pesquisas na base de dados da Coleção Scientific Electronic Library Online (SciELO) Brasil $^{11}$ e nos sítios eletrônicos das revistas Vigilância Sanitária em Debate: Sociedade, Ciência e Tecnologia (Visa em Debate) ${ }^{12}$ e Ciência \& Saúde Coletiva ${ }^{13}$. 
A Coleção SciELO Brasil é uma biblioteca eletrônica que disponibiliza textos completos de revistas científicas do Brasil de todas as áreas temáticas, que utilizam o procedimento de avaliação por pares dos artigos científicos que recebem ou encomendam para publicação ${ }^{14}$. Conta com 294 revistas permanentes de acesso aberto e em formato eletrônico ${ }^{15}$ distribuídas em nove grandes áreas temáticas: Ciências Agrárias, Biológicas, Saúde, Exatas e da Terra, Humanas, Sociais Aplicadas, Engenharias, Linguística, Letras e Artes e Psicanálise ${ }^{16}$.

A revista Visa em Debate, não indexada na Coleção SciELO Brasil, foi incluída nesta revisão por ser a única publicação específica em vigilância sanitária no Brasili ${ }^{17}$, cuja área da saúde pública tem sido fundamental no enfrentamento da COVID-19. O sítio eletrônico da revista Ciência \& Saúde Coletiva ${ }^{13}$ disponibiliza artigos na modalidade Ahead of Print - publicação de artigos que foram revisados por pares e aprovados e futuramente comporão uma edição a ser publicada -, o que justificou sua inclusão como mais uma fonte de dados nesta revisão.

A escolha dos descritores foi baseada no maior número de artigos científicos obtido em testes iniciais feitos na Coleção SciELO Brasil, partindo dos termos, em inglês, identificados nos Descritores em Ciências da Saúde: Wuhan coronavirus, coronavirus infections, 2019-nCoV, SARS-CoV-2, COVID-19 e pandemics. Um teste, que seguiu recomendações da literatura ${ }^{18}$, foi realizado utilizando o termo pandemics truncado com uma letra do alfabeto a esquerda (pandemic*). 0 refinamento foi realizado por meio de "tentativa e erro", comparando os resultados iniciais em relação ao maior número de artigos científicos obtidos nos testes, chegaram-se aos seguintes descritores: coronavirus, COVID-19 e pandemic.

A busca pelos artigos científicos na Coleção SciELO Brasil ocorreu por meio do emprego de técnicas de Web-scraping desenvolvidas e validadas especificamente para este fim. O Web-scraping permite a recuperação automatizada e direcionada de dados de interesse sobre determinado conteúdo disponível na internet, de forma rápida e resultando em ganho de escala ${ }^{19}$. A validação ocorreu com a conferência do número total de artigos obtido para cada descritor com as técnicas de Web-scraping e a busca feita manualmente na Coleção SciELO Brasil.

Ao pesquisar por descritor, de forma individual, em 2 de maio de 2020 (10 h $02 \mathrm{~min}$ ), o algoritmo desenhado, pelo autor PJGF, para mineração do conteúdo bibliográfico na Coleção SciELO Brasil reestruturou os dados de interesse apresentados para cada artigo identificado em um arquivo de valores separados por vírgulas (extensão csv). Neste algoritmo estavam incluídas, ainda, as técnicas de Web-scraping. Os dados recuperados e reestruturados foram: citação completa da publicação, autores, nome do periódico, data de publicação, número da edição da revista e resumos disponíveis. 0 arquivo foi convertido em formato compatível com o programa Microsoft Excel $®$, gerando o banco da mineração de texto a ser utilizado na fase subsequente desta revisão. Na Coleção SciELO Brasil não foi aplicado qualquer filtro de pesquisa.
Os três descritores no idioma português foram utilizados para a busca de artigos científicos no sítio eletrônico da revista Ciência \& Saúde Coletiva, realizada em $1^{\circ}$ de maio de 2020 (início: $16 \mathrm{~h}$ 26 min e término: $17 \mathrm{~h} 15 \mathrm{~min})^{13}$. A busca ocorreu conforme o filtro "tipo de artigo" disponível no sítio eletrônico da revista. No caso da revista Visa em Debate, as buscas ocorreram sem o uso de qualquer palavra-chave, observando as edições publicadas no período do estudo e, por conseguinte, artigos que abordassem o tema de interesse da revisão.

\section{Critérios de inclusão e exclusão}

Foram considerados elegíveis os estudos disponibilizados por revistas nacionais, entre $1^{\circ}$ de dezembro de 2019 e 2 de maio de 2020 (10 h $02 \mathrm{~min}$ ). A escolha desse intervalo coincidiu com o surgimento do primeiro caso de COVID-19 na cidade de Whuan (China) relatado na literatura científica ${ }^{1}$, concluindo após 154 dias, por ocasião do término da estratégia de busca. Nenhuma restrição de idioma e do tipo de artigo foi aplicada. 0 critério de inclusão foi: publicações, revisadas por pares, que abordaram qualquer assunto relacionado à COVID-19. Os critérios de exclusão foram: (1) assuntos de não interesse para a revisão; e (2) publicações de protocolos de pesquisa.

\section{Formação do banco de dados para a revisão de escopo}

O banco da mineração de texto sofreu alterações, com a inclusão e exclusão de variáveis, resultando na formação do banco de dados para esta revisão de escopo. As seguintes variáveis compuseram o referido banco: fonte de dados, descritores, citação completa do artigo, palavras-chave, país e instituição do primeiro autor, título, nome da revista, idioma de publicação, dia, mês e ano da publicação, objetivo do estudo, tipo de estudo/artigo e assunto abordado. A data de postagem foi utilizada para definir o dia e mês de publicação dos artigos que constavam nos sítios eletrônicos das revistas Visa em Debate e Ciência \& Saúde Coletiva.

Os resultados das buscas nos sítios eletrônicos das duas revistas foram registrados no banco de dados final. A afiliação institucional do primeiro autor dos artigos no sítio eletrônico da revista Ciência \& Saúde Coletiva foi obtida em pesquisa feita no Orcid do pesquisador, disponibilizado em cada manuscrito.

A classificação do tipo de estudo/artigo foi estabelecida de três maneiras: a) registrava-se o tipo de estudo definido pelo(s) autor(es); b) os artigos que não constavam essa definição foram classificados segundo o tipo de artigo enquadrado pela própria revista; e c) os resultados dos itens "a" e "b", ambos em uma única coluna da planilha, foram agrupados em sete categorias de pesquisas baseadas na literatura ${ }^{20,21,22,23}$ : i) pesquisa básica; ii) estudo analítico; iii) estudo descritivo; iv) pesquisa qualitativa; v) estudo de métodos; vi) revisão da literatura; e vii) comentário. Os artigos enquadrados no item "b", como editorial, opinião, debate e perspectivas, foram categorizados como "comentário".

A definição dos assuntos relacionados à COVID-19 explorados nos artigos ocorreram em duas fases. Na primeira, dois autores (DMM e LFL), de forma independente, fizeram leitura do texto, que 
resultou em uma primeira lista ampla de assuntos. Na segunda fase, as duas listas foram confrontadas durante videoconferência que teve dois objetivos: i) buscar consenso dos assuntos divergentes; e ii) agrupar os assuntos afins em macrotemas mais gerais.

\section{Seleção dos estudos}

Após a eliminação das duplicidades, os artigos registrados no banco de dados final, em elaboração, foram selecionados em duas etapas. Um dos autores (DMM) verificou o mês e ano de publicação dos artigos, visando exclusão daqueles que não se enquadraram no período da revisão. Na segunda etapa, esse mesmo autor se encarregou de examinar os títulos e/ou resumos das referências que continuaram no banco de dados final, em elaboração, após a finalização da primeira etapa, para eliminar os artigos, conforme os critérios de inclusão e exclusão. $\mathrm{O}$ arranjo final do banco de dados utilizado nesta revisão, com a inserção, em separado, das variáveis mencionadas anteriormente, ocorreu após a finalização dessas duas etapas.

\section{Extração e análise dos dados}

A extração dos dados baseou-se em processo iterativo, ou seja, os autores (DMM e LFL), de forma independente, se alternaram entre ler os resumos dos artigos incluídos na revisão e extrair as informações que complementassem as variáveis do banco de dados final, recorrendo, quando necessário, ao texto completo. Esse processo iterativo, também, foi realizado para a extração dos dados dos artigos científicos publicados na revista Visa em Debate $^{12}$ e disponibilizados no sítio eletrônico da revista Ciência \& Saúde Coletiva ${ }^{13}$. A extração dos dados realizada por dois pesquisadores visou garantir a correspondência dos dados finais registrados com aqueles extraídos das fontes de informações.

O banco de dados final foi submetido a uma análise quantitativa simples, usando estatística descritiva, como frequências e medidas de tendência central e dispersão, para fornecer resumos numéricos das características de interesse da literatura incluída na revisão. Os principais resultados das análises foram apresentados em formato de gráficos e tabelas alinhados ao objetivo e a pergunta de pesquisa. Informações sobre casos diagnosticados e óbitos por COVID-19, por data de confirmação no Brasil, foram obtidas em sítio eletrônico do Ministério da Saúde ${ }^{24}$.

Dados das revistas com artigos incluídos nesta revisão foram coletados em outra planilha, contemplando as seguintes variáveis: nome da revista, número do International Standard Serial Number (ISSN), subárea temática, número de edições publicadas a partir de $1^{\circ}$ de janeiro de 2020, última data de atualização da publicação/postagem e periodicidade da revista. Estes dados foram obtidos no sítio eletrônico da Coleção SciELO Brasil ${ }^{15}$ e revista Visa em Debate ${ }^{12}$. As modalidades do tipo Ahead of Print ou Fast Track e suplementos foram contados como edições publicadas pelas revistas.

Não foi necessária a apreciação por Comitê de Ética em Pesquisa devido à natureza do estudo e, também, os artigos científicos serem de domínio público.

\section{RESULTADOS}

Dados das buscas por artigos científicos

Foram Identificados 279 artigos científicos, dos quais $23(8,2 \%)$ foram excluídos como duplicados e 187 (67,0\%), por não se enquadrarem no período do estudo. A triagem de título, resumo e/ou texto completo foi conduzida para 69 artigos, sendo que $11(15,9 \%)$ foram excluídos, após aplicação dos critérios de inclusão e exclusão, resultando em $58(20,8 \%)$ artigos que compuseram esta revisão de escopo (Figura 1). Ressalta-se que 29 (50,0\%) artigos não apresentaram resumo em qualquer idioma.

A contribuição de cada fonte de informações com a quantidade de artigos científicos incluídos foi, nesta ordem: Coleção Brasil SciELO ( $n=44,75,9 \%)$; sítio eletrônico da revista Ciência \& Saúde Coletiva $(n=11,19,0 \%)$; e Visa em Debate $(n=3,5,1 \%)$. Do total de artigos selecionados, por meio do uso de descritores, para esta revisão $(n=55), 35(63,6 \%)$ foram identificados com o uso de apenas um deles. Ademais, 26 (44,8\%) artigos não continham qualquer palavra-chave, todos provenientes da Coleção SciELO Brasil.

\section{Dados sobre as revistas nacionais}

Durante o período do estudo, 22 revistas nacionais publicaram 58 artigos científicos sobre os mais variados assuntos relacionados à COVID-19. A mediana ficou em dois artigos disponibilizados por revista, variando de 1 a 11 . Todas as revistas pertenceram à grande área das Ciências da Saúde, com predomínio daquelas classificadas nas seguintes subáreas temáticas: Medicina ( $\mathrm{n}=12$ revistas), Saúde Coletiva/Saúde Pública ( $\mathrm{n}=3$ revistas) e Enfermagem e Odontologia, ambas com duas revistas. Do total de revistas permanentes na Coleção SciELO Brasil $(n=294)$, $21(7,1 \%)$ publicaram artigos de interesse desta revisão.

Dez revistas que adotam o modelo de publicação de fluxo contínuo, dispuseram $17(29,3 \%)$ artigos. Outras oito revistas que disponibilizaram uma modalidade de publicação chamada Ahead of Print/Fast Track, modalidades rápidas de publicação de artigos aprovados, foram responsáveis pela divulgação de 28 (48,3\%) artigos. Destas oito revistas, quatro delas mantinham a periodicidade bimestral de publicação dos seus artigos contra duas com publicação mensal.

Há predominância das revistas com periodicidade de publicação bimestral entre aquelas que apenas disponibilizam seus artigos nas edições planejadas para cada ano. A revista Ciência \& Saúde Coletiva apresentou o maior número de artigos científicos publicados ( $n=11,19,0 \%$ ) (Tabela 1 ). Todos os seus artigos estavam disponibilizados no sítio eletrônico da revista na modalidade Ahead of Print.

\section{Origem dos autores}

Dos 58 artigos incluídos na revisão, $51(88,0 \%)$ foram produzidos por autores brasileiros, três por portugueses e os demais cada um com um artigo, por autores norte-americano, italiano, 


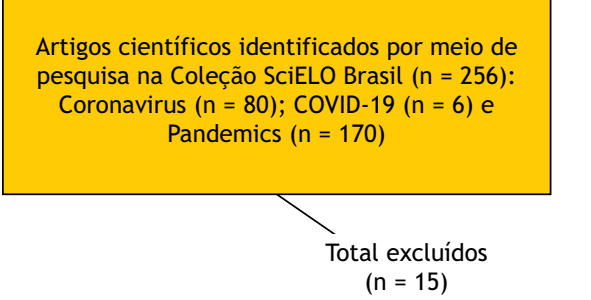

Artigos científicos identificados em outras fontes $(n=23)$ : - Visa em Debate $(n=3)$

- Sítio eletrônico da revista Ciência \& Saúde Coletiva $(n=20)$

COVID-19 $(n=12)$; Coronavirus $(n=0)$; e Pandemics $(n=8)$

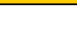

Total excluídos $(\mathrm{n}=8)$
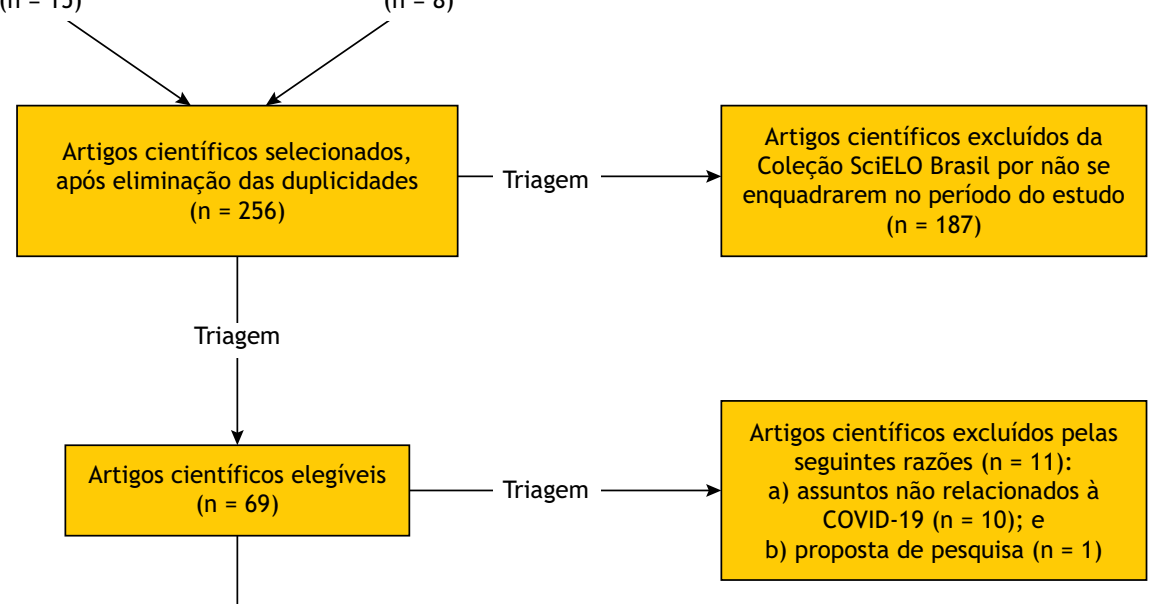

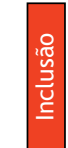

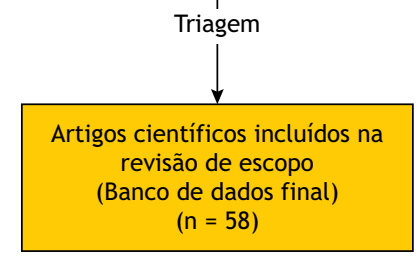

Fonte: Elaborada pelos autores, 2020.

Figura 1. Fluxograma do processo de identificação, seleção, elegibilidade e inclusão dos artigos científicos publicados por revistas nacionais entre $1^{\circ}$ de dezembro de 2019 e 2 de maio de 2020 (10 h 02 min).

espanhol e argentino. Os autores advêm de 37 instituições, as quais $27(73,0 \%)$ são de estabelecimentos de ensino superior e seis $(16,2 \%)$ estão ligadas às instituições hospitalares. A Universidade de São Paulo $(\mathrm{n}=7)$ registrou o maior número de artigos científicos, seguida da Fundação Oswaldo Cruz $(n=6)$, da Universidade Federal do Rio Grande do Sul, da Universidade Federal do Espírito Santo, do Hospital Israelita Albert Einstein e da Universidade Federal de São Paulo, cada um com três artigos.

\section{Caracterização dos artigos científicos}

Foram identificados 22 tipos de artigos/estudos, dos quais editorial ( $n=16,72,7 \%)$, estudo transversal $(n=5,22,7 \%)$ e opinião ( $n=5,22,7 \%$ ) foram os mais prevalentes (Tabela 2 ). Do total dos 58 artigos, $43(74,1 \%)$ foram caracterizados como tipo de artigo enquadrado pelas próprias revistas, ou seja, não constavam no texto, o tipo de estudo. A distribuição dos artigos com base nas sete categorias de pesquisa foi, nesta ordem: comentário $(n=43,74,1 \%)$, estudo descritivo $(n=8 ; 13,8 \%)$, revisão da literatura $(n=6,10,4 \%)$ e estudo analítico $(n=1,1,7 \%)$. Constatou-se apenas uma revisão sistemática ${ }^{25}$ e o estudo analítico publicado foi do tipo ecológico ${ }^{26}$. Especificamente, os sete artigos publicados em revistas nacionais por autores estrangeiros corresponderam a cinco comentários e dois estudos descritivos.
Não havia uma descrição explícita do objetivo em 23 (39,6\%) artigos, dos quais $13(56,5 \%)$ foram editoriais. A disponibilidade no idioma português foi identificada em 34 (58,6\%) artigos científicos. Destes, $18(53,0 \%)$ foram publicados em português/inglês. Já $24(41,4 \%)$ artigos foram publicados em inglês $(n=22)$ ou espanhol $(n=1)$ ou inglês/espanhol $(n=1)$.

A Figura 2 apresenta a distribuição dos 58 artigos no período estudado, cujo mês de abril concentrou $86,2 \%$ das publicações. O primeiro artigo foi publicado no dia 13 de março. Trata-se de artigo classificado como "comentário", publicado em português/inglês, que fez uma discussão geral sobre aspectos ligados à vigilância epidemiológica de casos, a estratégias de enfrentamento e a medidas de prevenção da COVID-19. 0 maior pico de publicações foi registrado no dia 9 de abril de 2020, com oito artigos científicos (Figura 2).

Foram encontradas quatro categorias de pesquisa, das sete previamente definidas, as quais abordaram 17 macrotemas relacionados à COVID-19. A categoria de pesquisa "comentário" teve representação em 16 dos 17 macrotemas tratados pelos artigos desta revisão (Tabela 2). Dos 58 artigos, "Isolamento social, saúde mental e outros aspectos relacionados a comportamentos sociais" foi o tema mais prevalente $(n=14,24,1 \%)$, seguido de 
Tabela 1. Revistas nacionais com publicação de artigos científicos sobre qualquer assunto relacionado à COVID-19 ( $\mathrm{n}=22$ revistas; $\mathrm{n}=58$ artigos científicos).

\begin{tabular}{|c|c|c|c|c|}
\hline Nome da revista & $\begin{array}{l}\text { Periodicidade de } \\
\text { publicação }\end{array}$ & $\mathrm{N}^{\circ}$ de edições* & $\mathrm{N}^{\circ}$ de artigos & $\begin{array}{l}\text { Data da última publicação } \\
\text { na Coleção SciELO Brasil }\end{array}$ \\
\hline \multicolumn{5}{|l|}{ Subárea temática: Saúde Coletiva/Saúde Pública $(n=3)$} \\
\hline - Ciência \& Saúde Coletiva & Mensal & 5 & 11 & $14 / 04 / 2020$ \\
\hline - Cadernos de Saúde Pública & Mensal & 5 & 8 & $28 / 04 / 2020$ \\
\hline - Revista de Saúde Pública & Fluxo contínuo & Não se aplica & 2 & $28 / 04 / 2020$ \\
\hline \multicolumn{5}{|l|}{ Subárea temática: Medicina $(n=12)$} \\
\hline - Brazilian Journal of Psychiatry & Bimestral & 3 & 6 & $28 / 04 / 2020$ \\
\hline - Clinics & Fluxo contínuo & Não se aplica & 4 & $28 / 04 / 2020$ \\
\hline - Arquivos Brasileiros de Cardiologia & Bimestral & 4 & 2 & $14 / 04 / 2020$ \\
\hline - Einstein & Fluxo contínuo & Não se aplica & 2 & $28 / 04 / 2020$ \\
\hline - International Journal of Cardiovascular Sciences & Bimestral & 3 & 2 & $24 / 04 / 2020$ \\
\hline - Radiologia Brasileira & Bimestral & 3 & 2 & $28 / 04 / 2020$ \\
\hline - Brazilian Journal of Cardiovascular Surgery & Bimestral & 3 & 1 & $28 / 04 / 2020$ \\
\hline - Jornal Brasileiro de Pneumologia & Bimestral & 5 & 1 & $17 / 04 / 2020$ \\
\hline - Revista do Colégio Brasileiro de Cirurgiões & Fluxo contínuo & Não se aplica & 1 & $07 / 04 / 2020$ \\
\hline - Revista Paulista de Pediatria & Fluxo contínuo & Não se aplica & 1 & $28 / 04 / 2020$ \\
\hline - Revista da Sociedade Brasileira de Medicina Tropical & Fluxo contínuo & Não se aplica & 1 & $28 / 04 / 2020$ \\
\hline - Trends in Psychiatry and Psychotherapy & Trimestral & 2 & 1 & $14 / 04 / 2020$ \\
\hline \multicolumn{5}{|l|}{ Subárea temática: Epidemiologia e Saúde Pública (n = 2) } \\
\hline - Epidemiologia e Serviços de Saúde & Trimestral & 2 & 3 & $24 / 04 / 2020$ \\
\hline - Revista Brasileira de Epidemiologia & Fluxo contínuo & Não se aplica & 3 & $17 / 04 / 2020$ \\
\hline \multicolumn{5}{|l|}{ Subárea temática: Vigilância Sanitária e Saúde Pública $(n=1)$} \\
\hline - Visa em Debate & Trimestral & 2 & 3 & $24 / 04 / 2020^{* *}$ \\
\hline \multicolumn{5}{|l|}{ Subárea temática: Odontologia $(\mathrm{n}=2)$} \\
\hline - Pesquisa Brasileira em Odontopediatria e Clínica Integrada & Fluxo contínuo & Não se aplica & 1 & $24 / 04 / 2020$ \\
\hline - RGO - Revista Gaúcha de Odontologia & Fluxo contínuo & Não se aplica & 1 & $17 / 04 / 2020$ \\
\hline \multicolumn{5}{|l|}{ Subárea temática: Enfermagem ( $\mathrm{n}=2$ ) } \\
\hline - Revista Brasileira de Enfermagem & Bimestral & 3 & 1 & $24 / 04 / 2020$ \\
\hline - Revista Latino-Americana de Enfermagem & Fluxo contínuo & Não se aplica & 1 & $17 / 04 / 2020$ \\
\hline
\end{tabular}

Fonte: Elaborada pelos autores, 2020.

* O número de edições das revistas foi contado a partir de $1^{\circ}$ de janeiro de 2020; ** Data que constava no sítio eletrônico da revista, modalidade Fast Track [Pré-prova COVID-19 (SARS-CoV-2)]. A revista Visa em Debate não faz parte da Coleção SciELO Brasil.

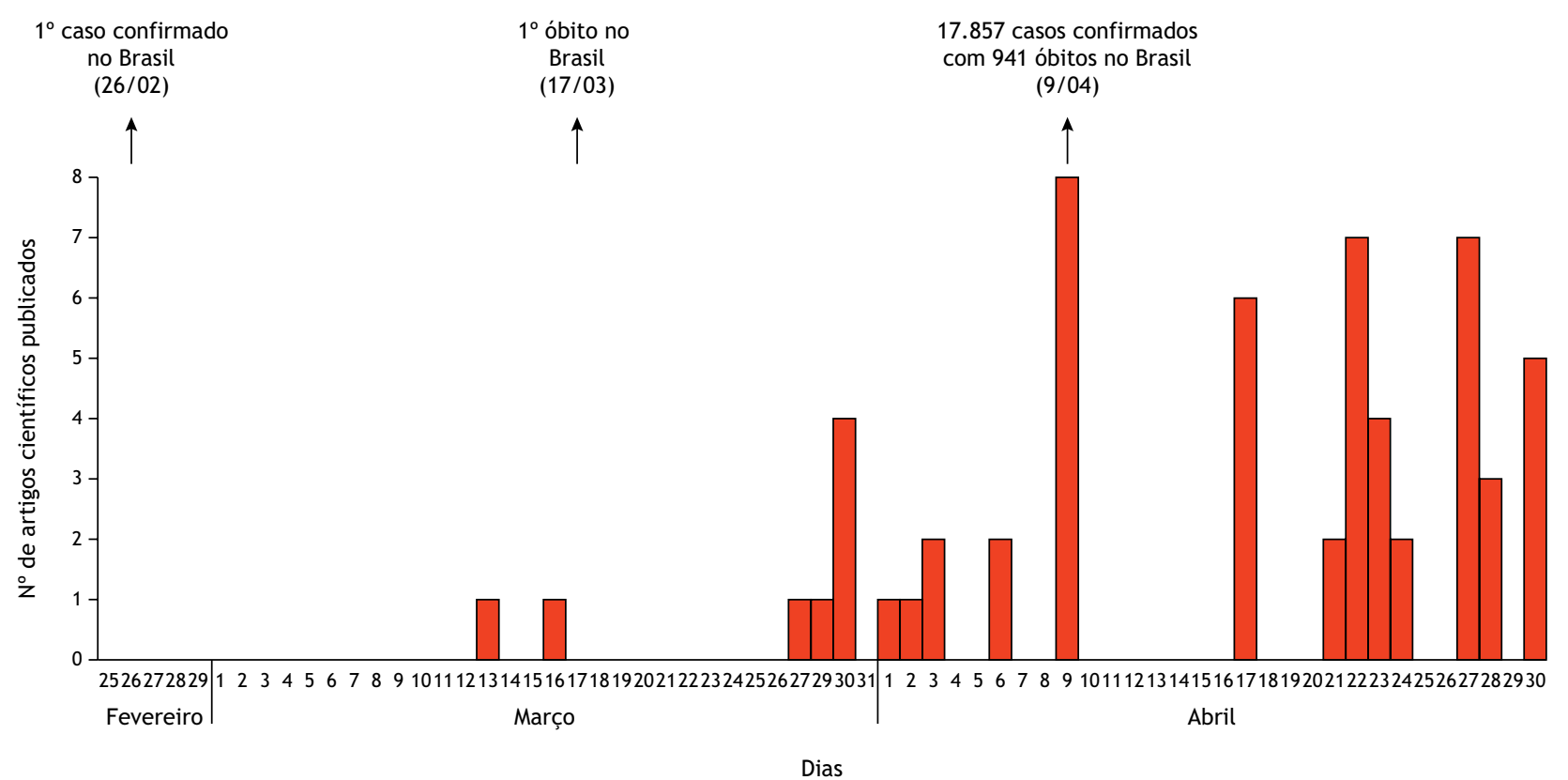

Fonte: Elaborada pelos autores, 2020.

Figura 2. Recorte temporal da distribuição diária dos artigos publicados no período de $1^{\circ}$ de dezembro de 2019 a 2 de maio de 2020 (10 h 02 min) ( $\mathrm{n}=58$ ). A figura apresenta o resultado da pesquisa no período entre 25 de fevereiro e 30 de abril de 2020 . Não houve registros de artigos publicados nos dias $1^{\circ}$ e 2 de maio. 
“medidas de prevenção, controle e respostas" ( $\mathrm{n}=7,12,1 \%)$, "manejo de outras enfermidades" ( $n=6,10,3 \%)$ e "produção e uso de evidências científicas” ( $n=5,8,6 \%$ ) (Tabela 2 ).

O estudo analítico fez uma análise da distribuição geográfica de casos da COVID-19 e de leitos de terapia intensiva exclusivos para a doença no estado do Ceará. Os autores concluíram que a interiorização da doença demandaria medidas de contingência voltadas à distribuição dos leitos hospitalares específicos para atender os casos de COVID-19 no Ceará26.

O estudo de revisão sistemática, baseado nas diretrizes do PRISMA, buscou avaliar a melhor estratégia para métodos de desinfecção de chupetas no contexto da COVID-19. Os autores concluíram que, devido aos diversos métodos de desinfecção de chupetas e aos diferentes níveis de acessibilidade aos agentes desinfetantes, permanece incerto um consenso sobre a descontaminação de chupetas ${ }^{25}$.

Os oitos estudos descritivos podem ser divididos em três tipos de assuntos. Dois estudos abordaram diagnósticos por imagem, utilizando a tomografia ${ }^{27,28}$. Um estudo sobre vigilância epidemiológica de casos em embarcação no porto de Santos (São Paulo) ${ }^{29}$. Cinco estudos abordaram aspectos comportamentais, crenças e percepção frente à pandemia da COVID-19 $9^{30,31,32,33,34}$.

O último estudo analisou os níveis de estresse, ansiedade e depressão desde a introdução do vírus e os níveis de sintomas psicológicos, segundo idade, comorbidades e confinamento em amostra da população do País Basco (norte da Espanha) ${ }^{34}$. Os resultados desse estudo mostraram que, ainda que os níveis de sintomas tenham sido baixos no início do confinamento, os indivíduos mais jovens e com comorbidades referiram mais sintomas que o restante da população. Foi detectado, também, maior nível de sintomas desde o confinamento, quando a população foi proibida de sair de suas casas $^{34}$.

Com relação à categoria de pesquisa "comentário", alguns artigos científicos abordaram as consequências da COVID-19 para a saúde mental e para aspectos comportamentais, como aumento do risco de suicídio ${ }^{35}$ e riscos para violências autoinflingidas ${ }^{36}$. Outras enfermidades que foram retratadas nos artigos focaram mais a relação da COVID-19 com questões ligadas ao coração ${ }^{37,38,39}$. Considerações sobre o uso de evidências científicas ${ }^{40}$ e a importância das publicações científicas em tempos de crise pandêmica ${ }^{41,42}$ foram outros assuntos, também, encontrados nessa categoria de pesquisa.

\section{DISCUSSÃO}

Esta revisão de escopo mapeou revistas científicas no Brasil que publicaram artigos sobre assuntos relacionados à COVID-19 entre $1^{\circ}$ de dezembro de 2019 e 2 de maio de 2020.

Embora o número acumulado de artigos tenha aumentado, observou-se lacunas importantes sobre tipos de estudo, grandes áreas temáticas e macrotemas. Particularmente, ensaios clínicos randomizados e estudos de coorte não foram identificados até 2 de maio ( $10 \mathrm{~h} 02 \mathrm{~min}$ ). Estudos da pesquisa básica e qualitativa também não foram encontrados nesta revisão. Todas as revistas pertenciam à grande área temática das Ciências da Saúde. Estudos sobre modelos matemáticos para a predição do

Tabela 2. Mapa de macrotemas relacionados à COVID-19, segundo cada categoria de pesquisa definida para os artigos científicos $(\mathrm{n}=58)$.

\begin{tabular}{|c|c|c|c|c|}
\hline \multirow{2}{*}{$\begin{array}{l}\text { Macrotemas } \\
(n=17)\end{array}$} & \multicolumn{4}{|c|}{ Categoria de pesquisa $(n=4)$} \\
\hline & $\begin{array}{l}\text { Estudo analítico } \\
\text { (n) }\end{array}$ & $\begin{array}{l}\text { Estudo descritivo } \\
\text { (n) }\end{array}$ & $\begin{array}{l}\text { Revisão da literatura } \\
\text { (n) }\end{array}$ & $\begin{array}{l}\text { Comentário } \\
(n)\end{array}$ \\
\hline $\begin{array}{l}\text { Isolamento social, saúde mental e outros aspectos relacionados a } \\
\text { comportamentos sociais }\end{array}$ & 0 & 5 & 1 & 8 \\
\hline Medidas de prevenção, controle e respostas & 0 & 0 & 1 & 6 \\
\hline Manejo de outras enfermidades & 0 & 0 & 1 & 5 \\
\hline Produção e uso de evidências científicas & 0 & 0 & 0 & 5 \\
\hline Estratégias de diagnóstico & 0 & 2 & 0 & 2 \\
\hline Vigilância epidemiológica de casos & 0 & 1 & 0 & 3 \\
\hline Política de enfrentamento da pandemia e alocação de recursos em saúde & 1 & 0 & 0 & 4 \\
\hline Aspectos clínicos e manejo da COVID-19 & 0 & 0 & 2 & 1 \\
\hline Violência doméstica & 0 & 0 & 0 & 2 \\
\hline Educação em saúde & 0 & 0 & 0 & 1 \\
\hline Fundamentos histórico-sociais da pandemia de COVID-19 & 0 & 0 & 0 & 1 \\
\hline Alta transmissibilidade da doença em consultórios dentários & 0 & 0 & 0 & 1 \\
\hline Segurança alimentar & 0 & 0 & 0 & 1 \\
\hline Métodos de desinfecção de chupetas & 0 & 0 & 1 & 0 \\
\hline Atividade física & 0 & 0 & 0 & 1 \\
\hline Modelos de governança de dados e questões ético-legais & 0 & 0 & 0 & 1 \\
\hline Fake News & 0 & 0 & 0 & 1 \\
\hline Total (n) & 1 & 8 & 6 & 43 \\
\hline
\end{tabular}

Fonte: Elaborada pelos autores, 2020.

(n): frequência absoluta. 
comportamento da doença, desenvolvimento de testes de diagnósticos, medicamentos, vacinas, entre outros, para enfrentamento da COVID-19 e estudos econômicos sobre o impacto de medidas adotadas para o controle da doença são exemplos de importantes macrotemas não identificados nesta revisão.

As lacunas podem ser explicadas pela rápida propagação geográfica do surto e compreensão limitada do novo vírus e da COVID-1943. Além disso, alguns tipos de estudo são demorados, como os ensaios clínicos randomizados, que levam em média de 5,5 anos desde o início até sua publicação ${ }^{44}$. Pesquisadores brasileiros e de outros países costumam publicar seus estudos com potencial relevância científica em revistas internacionais de alta credibilidade e impacto acadêmico ${ }^{45,46}$. Assim, quanto maior o ranking da revista no meio acadêmico onde o pesquisador publica, maior prestígio terá em sua comunidade, bem como aumenta suas chances de conseguir uma posição em uma instituição de excelência ou ao menos de destaque ${ }^{46}$.

Outra razão da lacuna pode estar relacionada a diferenças na cobertura das revistas por grandes áreas temáticas na Coleção SciELO Brasil, justificando, em parte, a presença somente de revistas das Ciências da Saúde. Por exemplo: a cobertura relativa da SciELO favorece as revistas das Ciências da Saúde, Humanas e Sociais Aplicadas ${ }^{45}$.

Nas estratégias de busca desta revisão não foi aplicado qualquer tipo de filtro relacionado à restrição de tempo. Isso justifica a exclusão de mais da metade dos artigos científicos. Por exemplo, para o descritor "pandemic", que apresentou o maior número de artigos identificados, foram excluídos 127 (74,7\%), do total de 170 capturado na Coleção SciELO Brasil. No banco de dados inicial resultante havia um artigo de 1993, publicado pela revista Physis: Revista de Saúde Coletiva ${ }^{47}$, e um outro de outubro de 2019, disponibilizado na revista Arquivos de Gastroenterologia ${ }^{48}$.

O maior número de artigos disponibilizado proveio das revistas que desenvolveram modalidades rápidas de publicação (Ahead of Print/Fast Track), garantindo ainda a revisão por pares, ou vinham adotando um modelo de publicação de fluxo contínuo. A publicação em revistas acadêmicas revisadas por pares, geralmente, implica longos atrasos entre o envio do artigo e sua publicação ${ }^{49}$. Os atrasos podem comprometer os avanços da ciência ${ }^{49}$ e, em situações pandêmicas como da COVID-19, prejudicarem na redução de sofrimentos e vidas humanas salvas.

Estudos têm apontado que a modalidade de pré-impressões (Preprints), artigos publicados abertamente online antes da revisão por pares, oferece uma oportunidade para acelerar a disseminação de descobertas científicas e para apoiar as respostas a surtos de doenças infecciosas ${ }^{50}$. As pré-impressões publicadas durante os surtos de Ebola e Zika ficaram disponíveis, após revisadas por pares, mais de 100 dias depois ${ }^{50}$. Embora esta revisão de escopo não tenha contabilizada as pré-impressões de artigos, a SciELO aderiu a essa modalidade ${ }^{51}$.

Uma revisão de escopo sobre a produção científica internacional $(n=249)$, entre $1^{\circ}$ de dezembro de 2019 e 6 de fevereiro de
2020, demonstrou que a maioria ( $\mathrm{n}=192,77,1 \%)$ dos artigos foi publicada em revistas revisadas por pares ${ }^{43}$. No Brasil, até aquela data, inexistiam estudos publicados sobre a COVID-19 em revistas nacionais disponíveis nas fontes de informação utilizadas nesta revisão de escopo. O primeiro artigo publicado ocorreu após 16 dias do primeiro caso confirmado no Brasil. Em 17 de março, data do registro do primeiro óbito no país, havia apenas dois artigos disponíveis sobre o tema.

A publicação em revistas nacionais de artigos em outro idioma, que não o português, pode ser uma barreira importante no acesso a informações científicas qualificadas em tempos de COVID-19 para milhares de brasileiros com limitado conhecimento da língua inglesa?.

A busca por maior internacionalização tem crescido o número de revistas nacionais que somente aceitam artigos publicados em inglês ou exigem que ao menos uma das versões esteja nesse idioma ${ }^{46}$. Esse fenômeno pode levar a população leiga a buscar informação em fontes nem sempre confiáveis. Redes sociais têm se tornado cada vez mais uma fonte de informação amplamente aceita pela população em geral, aumentando com isso a disseminação de informações falsas, mas de rápido acesso e de fácil compreensão. Algo que deverá ser levado em consideração pelos cientistas locais no decorrer da pandemia.

Ensaio clínico randomizado, estudo de coorte e pesquisa básica não foram encontrados na produção científica nacional sobre assuntos relacionados à COVID-19. Esses achados apresentaram semelhanças e diferenças em relação à revisão de escopo publicada por Lv et al. ${ }^{43}$. Estes autores também não identificaram ensaio clínico randomizado e estudo de corte ${ }^{43}$. Por outro lado, artigos sobre pesquisa básica foram encontrados na revisão dos pesquisadores. Além disso, artigos de pesquisa qualitativa não foram identificados em nosso estudo, apesar da cobertura relativa da SciELO favorecer as revistas das Ciências da Saúde, Humanas e Sociais Aplicadas ${ }^{45}$.

Não ocorreu uma concentração de macrotemas sobre a COVID19 abordados pelos artigos desta revisão. Lv et al. ${ }^{43}$ encontraram resultados semelhantes na sua revisão de escopo, sendo que "prevenção e controle" foi o tema mais prevalente ${ }^{43}$. Diferentemente do que foi mencionado por Ornell et al. ${ }^{52}$ que, durante as pandemias, tendem a ser subestimadas e negligenciadas as implicações psicológicas e psiquiátricas secundárias da doença, nossa revisão registrou um maior número de artigos que trataram sobre macrotemas ligados ao "Isolamento social, saúde mental e outros aspectos relacionados a comportamentos sociais" seguido de "medidas de prevenção, controle e respostas". "Saúde mental" ocupou a $15^{\mathrm{a}}$ colocação entre os macrotemas abordados pelos artigos do estudo de Lv et al. ${ }^{43}$.

Os resultados desta revisão precisam ser considerados à luz das suas limitações. Apesar dos autores terem planejado cuidadosamente as estratégias de buscas, consultando Descritores em Ciências da Saúde ${ }^{53}$ e realizando testes com diferentes descritores (um deles truncado ${ }^{18}$ ), inclusive alguns utilizados por outros autores $^{43}$, para escolher aqueles mais apropriados, existe a 
possibilidade de que artigos científicos publicados por revistas nacionais, mesmo aquelas da Coleção SciELO Brasil, não tenham sido identificados nesta revisão. No entanto, é razoável esperar que esta revisão de escopo, até o dia 2 de maio de 2020, tenha abrangido a maior parte dos artigos sobre assuntos ligados à COVID-19 revisados por pares, disponíveis na Coleção SciELO Brasil e todos aqueles disponibilizados pelas revistas Visa em Debate e Ciência \& Saúde Coletiva.

A validação do algoritmo de mineração de textos, em termos de número de artigos encontrados na Coleção SciELO Brasil, com buscas feitas, de forma manual, foi outro cuidado que os autores tiveram neste estudo. Ademais, o uso de descritores em inglês, isoladamente, atualizados e mais gerais, como "coronavirus" e "pandemic", pode ter melhorado a qualidade da busca pelos artigos de interesse da revisão.

É provável, ainda, que este estudo apresente elementos de subjetividade na definição e agrupamento dos assuntos abordados pelos diversos artigos. No entanto, a estratégia planejada e descrita na seção "Método", como a classificação dos assuntos feita por dois autores, de forma independente, e, em seguida, o confrontamento das duas listas para defini-los e agrupá-los, por consenso, em termos mais gerais pode ter reduzido a subjetividade.

Esta revisão de escopo tem pontos positivos que merecem ser destacados. Foi realizada uma pesquisa sistemática baseada em referências reconhecidas internacionalmente, como PRISMA-SCR ${ }^{9}$ e Joanna Briggs Institute Reviewer's Manual ${ }^{10}$. Utilizou-se técnicas de mineração de textos para identificação dos artigos científicos que geraram um ganho de escala muito importante para a finalização desta revisão em um tempo razoavelmente curto. Foi feito uso de uma fonte de dados de abrangência nacional, ampla cobertura de áreas temáticas e rigor na indexação de revistas. A busca por artigos científicos também incluiu os sítios eletrônicos de duas importantes revistas na área da saúde pública e vigilância sanitária. Ademais, o número de artigos científicos na revisão é suficientemente razoável para ilustrar a produção científica nacional sobre macrotemas relacionados à COVID-19 no período estudado, que também poderá ser útil em direcionar editais de órgãos/agências de fomento para financiamento de pesquisas científicas no Brasil.

\section{CONCLUSÕES}

Esta revisão de escopo apresentou um mapa da produção científica sobre qualquer assunto relacionado à COVID-19 no Brasil a partir de revistas nacionais que disponibilizaram, de forma eletrônica, artigos, entre $1^{\circ}$ de dezembro de 2019 e 2 de maio de 2020 (10 h 02 min).

Nesta produção há lacunas importantes, principalmente no que tange aos ensaios clínicos randomizados e estudos de coorte, que precisam ser preenchidas com a realização de pesquisas. É essencial, também, ampliar o número de revisões sistemáticas e de estudos sobre impacto socioeconômico de medidas adotadas para prevenção e controle da doença, visando contar com evidências de qualidade publicadas em português. Outras grandes áreas temáticas devem ser estimuladas a desenvolver e a publicar suas pesquisas em revistas nacionais, a exemplo, das Ciências Sociais Aplicadas e Ciências Exatas e da Terra. Neste último caso, com o desenvolvimento de estudos sobre modelos matemáticos para a predição da propagação da COVID-19 nas diferentes regiões do país.

Neste momento de epidemia no Brasil, a produção científica nacional sobre a COVID-19 tem papel imediato na formulação de políticas públicas de enfrentamento da doença e na orientação de decisões clínicas ${ }^{42}$, no que tange as ações de prevenção e tratamento, incluindo a elaboração de Protocolos Clínicos e Diretrizes Terapêuticas ${ }^{40}$, cabendo às universidades brasileiras o papel de protagonistas nessa produção.

\section{REFERÊNCIAS}

1. Huang C, Wang $Y$, Li X, Ren L, Zhao J, Hu $Y$ et al. Clinical features of patients infected with 2019 novel coronavirus in Wuhan, China. Lancet. 395(10223):497-506. https://doi.org/10.1016/S0140-6736(20)30183-5

2. Representação da Organização Panamericana da Saúde no Brasil - OPAS Brasil. COVID-19 (doença causada pelo novo coronavírus). Folha informativa. 30 abr 2020[acesso 1 maio 2020]. Disponível em: https: / / www.paho.org/bra/index.php?option=com_ content\&view $=$ article\&id $=6101$ : covid 19 \& Itemid $=875$

3. John Hopkins University - JHU. COVID-19 dashboard by the center for systems science and engineering (CSSE) at Johns Hopkins university. Baltimore: John Hopkins University; 2020[acesso 6 maio 2020]. Disponível em: https: //coronavirus.jhu.edu/map.html

4. Ministério da Saúde (BR). Boletim epidemiológico COECOVID-19. Brasília: Ministério da Saúde; 2020[acesso 1 maio
2020]. Disponível em: https://portalarquivos.saude.gov. $\mathrm{br} /$ images/pdf/2020/April/27/2020-04-27-18-05h-BEE14Boletim-do-COE.pdf

5. Sharma M, Scarr S, Kelland K. Coronavirus and the risks of speed science. World Economic Forum Agenda. 24 mar 2020[acesso 1 maio 2020]. Disponível em: https://www.weforum.org/agenda/2020/03/ speed-science-coronavirus-covid19-research-academic/

6. Machado R. Desigualdade no acesso à informação e tecnologias é o desafio à imersão profunda do Brasil na agricultura 4.0: entrevista especial com Silvia Maria Massruhá. São Leopoldo: Instituto Humanitas Unisinos; 2020[acesso 1 maio 2020]. Disponível em: http: / /www.ihu. unisinos.br/159-noticias/entrevistas/596142-desigualdadeno-acesso-a-informacao-e-tecnologias-e-o-desafio-aimersao-profunda-do-brasil-a-agricultura-4-0-entrevistaespecial-com-silvia-maria-massruha 
7. British Council - BC. Learning english in Brazil understanding the aims and expectations of the brazilian emerging middle classes. São Paulo: British Concil; 2014[acesso 2 maio 2020]. Disponível em: https://www.britishcouncil.org.br/sites/ default/files/learning_english_in_brazil.pdf

8. Munn Z, Peters MDJ, Stern C, Tufanaru C, McArthur A, Aromataris E. Systematic review or scoping review: guidance for authors when choosing between a systematic or scoping review approach. BMC Med Res Methodol. 2018;18:1-7. https://doi.org/10.1186/s12874-018-0611-x

9. Tricco AC, Lillie E, Zarin W, O’Brien KK, Colquhoun H, Levac $D$ et al. Prisma extension for scoping reviews (Prisma-ScR): checklist and explanation. Ann Intern Med. 2018;169(7):467-73. https://doi.org/10.7326/M18-0850

10. Peters MDJ, Godfrey C, McInerney P, Munn Z, Tricco AC, Khalil H. Scoping reviews. In: Aromataris E, Munn Z, editors. Joanna Briggs institute reviewer's manual. Adelaide: Joanna Briggs Institute; 2020[acesso 6 maio 2020]. Disponível em: https://wiki.joannabriggs.org/display/MANUAL/ Chapter+11\%3A+Scoping+reviews

11. Scientific Electronic Library Online - SciELO. Home. São Paulo: Scientific Electronic Library Online; 2008[Acesso 2 maio 2020]. Disponível em: https://www.scielo.br/scielo. php?script=sci_home\&tng=pt\&nrm=iso

12. Fundação Oswaldo Cruz - Fiocruz. Vigilância sanitária em debate: sociedade, ciência \& tecnologia: edições anteriores. Rio de Janeiro: Fundação Oswaldo Cruz; 2013[acesso 2 maio 2020]. Disponível em: https://visaemdebate.incqs. fiocruz.br/index.php/ visaemdebate/issue/archive

13. Associação Brasileira de Saúde Coletiva - Abrasco. Revista ciência \& saúde coletiva: ahead of print. Rio de Janeiro: Associação Brasileira de Saúde Coletiva; 1996[acesso 1 maio 2020]. Disponível em: http://www.cienciaesaudecoletiva. com.br/ahead-of-print

14. Scientific Electronic Library Online - SciELO. Critérios, política e procedimentos para a admissão e a permanência de periódicos científicos na coleção SciELO Brasil. São Paulo: Scientific Electronic Library Online; 2008[acesso 3 maio 2020]. Disponível em: https: / /www.scielo.br/ avaliacao/20141003NovosCriterios_SciELO_Brasil.pdf

15. Scientific Electronic Library Online - SciELO. Coleção da biblioteca. São Paulo: Scientific Electronic Library Online; 2008[acesso 1 maio 2020]. Disponível em: https://www. scielo.br/scielo.php?script=sci_alphabetic\&lng=pt\&nrm=iso

16. Scientific Electronic Library Online - SciELO. Periódicos: lista de periódicos por assunto. São Paulo: Scientific Electronic Library Online; 2008[acesso 3 maio 2020]. Disponível em: https://scielo.org/pt

17. Agência Nacional de Vigilância Sanitária - Anvisa. Anvisa lança edital para seleção de artigos científicos. Visa em Debate. 26 nov 2019[acesso 1 maio 2020]. Disponível em: http://portal.anvisa.gov.br/noticias/-/asset_publisher/ FXrpx9qY7FbU/content/anvisa-divulga-edital-paraselecao-de-artigos-cientificos/219201/pop_up?_101_ INSTANCE_FXrpx9qY7FbU_viewMode=print\&_101_INSTANCE_ FXrpx9qY7FbU_languageld=pt_BR

18. Salvador-Oliván JA, Marco-Cuenca G, Arquero-Avilés R. Errors in search strategies used in systematic reviews and their effects on information retrieval. J Med Libr Assoc. 2019;107(2):210-21. https://doi.org/10.5195/jmla.2019.567

19. Meschenmoser P, Meuschke N, Hotz M, Gipp B. Scraping scientific web repositories: challenges and solutions for automated content extraction. D Lib Mag. 2016;22(9-10):1-7. https://doi.org/10.1045/september2016-meschenmoser

20. Institute for Quality and Efficiency in Health Care IQWiG. What types of studies are there? NCBI Bookshelf. 15 jun 2016[acesso 6 maio 2020]. Disponível em: https://www.ncbi.nlm.nih.gov/books/NBK390304/

21. Lamorte WW. What is a scientific article? In: Lamorte WW. Overview of critical reading: how to critically review an article. Boston: Boston University School of Public Health; 2016[acesso 6 maio 2020]. Disponível em: http://sphweb.bumc.bu.edu/otlt/MPH-Modules/EP/EP713_ CriticalReview/EP713_CriticalReview2.html\#headingtaglink_1

22. Röhrig B, Prel JB, Wachtlin D, Blettner M. Types of study in medical research. Dtsch Arztebl Int. 2009;106(15):262-8. https://doi.org/10.3238/arztebl.2009.0262

23. Pai $M$, Filion $\mathrm{K}$. Classification of study designs (version 8 ). Montreal: McGill University; 2016[acesso 6 maio 2020]. Disponível em: https://www.teachepi.org/wp-content/ uploads/OldTE/documents/courses/Classification\%20Design.pdf

24. Ministério da Saúde (BR). Painel coronavírus. Coronavírus Brasil. 6 maio 2020[acesso 6 maio 2020]. Disponível em: https://covid.saude.gov.br/

25. Souza VGC, Lopes DF, Machado FC, Fabri RL, Apolônio ACM, Souza VGC et al. The novel coronavirus: an alert for pacifiers' disinfection. Pesqui Bras Odontopediatria Clin Integr. 2020;20:1-11. https://doi.org/10.1590/pboci.2020.070

26. Pedrosa NL, Albuquerque NLS. Análise espacial dos casos de COVID-19 e leitos de terapia intensiva no estado do Ceará, Brasil. Cienc Saude Coletiva. 2020:1-19. https://doi.org/10.1590/SciELOPreprints. 181

27. Barbosa PNVP, Bitencourt AGV, Miranda GD, Almeida MFA, Chojniak R. Chest CT accuracy in the diagnosis of SARS-CoV-2 infection: initial experience in a cancer center. Radiol Bras. 2020:1-5. https://doi.org/10.1590/0100-3984.2020.0040.

28. Chate RC, Fonseca EKUN, Passos RBD, Teles GBS, Shoji H, Szarf G. Presentation of pulmonary infection on CT in COVID-19: initial experience in Brazil. J Bras Pneumol. 2020;46(2):1-4. https: //doi.org/10.36416/1806-3756/e20200121

29. Fernandes EG, Santos JS, Sato HK. Outbreak investigation in cargo ship in times of COVID-19 crisis, port of Santos, Brazil. Rev Saude Publica. 2020;54:1-4. https://doi.org/10.11606/s1518-8787.2020054002461

30. Lima DLF, Dias AA, Rabelo RS, Cruz ID, Costa SC, Nigri FMN et al. COVID-19 no estado do Ceará: comportamentos e crenças na chegada da pandemia. Cien Saude Colet. 2020;25(5):1575-86. https: / / doi.org/10.1590/1413-81232020255.07192020

31. Bezerra A, Silva CEM, Soares FRG, Silva JAM. Fatores associados ao comportamento da população durante $o$ isolamento social na pandemia de COVID-19. Cien Saude Colet. 2020:1-24. https://doi.org/10.1590/SciELOPreprints.123

32. Johnson MC, Saletti-Cuesta L, Tumas N. Emociones, preocupaciones y reflexiones frente a la pandemia del COVID-19 en Argentina. Cien Saude Colet. 2020. 
33. Carvalho LF, Pianowski G, Gonçalves AP. Personality differences and COVID-19: are extroversion and conscientiousness personality traits associated with engagement with containment measures? Trends Psychiatry Psychother. 2020:1-6. https: / /doi.org/10.1590/2237-6089-2020-0029

34. Ozamiz-Etxebarria N, Dosil-Santamaria M, Picaza-Gorrochategui $M$, Idoiaga-Mondragon $N$. Niveles de estrés, ansiedad y depresión en la primera fase del brote del COVID-19 en una muestra recogida en el norte de España. Cad Saude Publica. 2020;36(4):1-9. https://doi.org/10.1590/0102-311×00054020

35. Sher L. An infectious disease pandemic and increased suicide risk. Braz J Psychiatry. 2020;42(3):239-40. https://doi.org/10.1590/1516-4446-2020-0989

36. Deslandes SF, Coutinho T. O uso intensivo da internet por crianças e adolescentes no contexto da COVID-19 e os riscos para violências autoinflingidas. Cien Saude Coletiva. 2020.

37. Oliveira GMM, Pinto FJ. COVID-19: a matter close to the heart. Int J Cardiovasc Sci. 2020:1-4. https://doi.org/10.36660/ijcs.20200057

38. Strabelli TMV, Uip DE. COVID-19 e o coração. Arq Bras Cardiol. 2020;114(4):598-600. https://doi.org/10.36660/abc.20200209

39. Del Giglio M, Tamagnini G. Cardiac surgery in the time of coronavirus. Braz J Cardiovasc Surg. 2020:1-2. https: / / doi.org/10.21470/1678-9741-2020-0161

40. Mota DM, Kuchenbecker RS. Considerações sobre o uso de evidências científicas em tempos de pandemia: o caso da COVID-19. Vigil Sanit Debate. 2020;8(2):1-19. https://doi.org/10.22239/2317-269x.01541

41. Moreira LFP. The importance of scientific publications in times of pandemic crisis. Clinics. 2020;75:1. https://doi.org/10.6061/clinics/2020/e1895

42. Carvalho MS, Lima LD, Coeli CM. Ciência em tempos de pandemia. Cad Saude Publica. 2020;36(4):1-3. https://doi.org/10.1590/0102-311 x0055520

43. Lv M, Luo X, Estill J, Liu Y, Ren M, Wang $J$ et al. Coronavirus disease (COVID-19): a scoping review. Euro Surveill. 2020;25(15):1-13. https://doi.org/10.2807/1560-7917.ES.2020.25.15.2000125

44. Ioannidis JP. Effect of the statistical significance of results on the time to completion and publication of randomized efficacy trials. JAMA. 1998;279(4):281-6. https://doi.org/10.1001/jama.279.4.281

45. Packer AL. The emergence of journals of Brazil and scenarios for their future. Educ Pesqui. 2014;40(2):301-23. https://doi.org/10.1590/S1517-97022014061860

46. Alcadipani R. Periódicos brasileiro em inglês: a mímica do publish or perish global. Rev Adm Empres. 2017;57(4):405-11. https://doi.org/10.1590/s0034-759020170410

47. Santos LAC, Moraes C, Coelho VSP. A politização do sangue no primeiro mundo. Physis. 1993;3(2):165-92. https://doi.org/10.1590/S0103-73311993000200007

48. Linden MA, Freitas RGBON, Hessel G, Marmo DB, Bellomo-Brandão MA. Definition of vitamin d deficiency in schoolchildren: systematic review with meta-analysis. Arq Gastroenterol. 2019;56(4):425-30. https://doi.org/10.1590/s0004-2803.201900000-64

49. Björk BC, Solomon D. The publishing delay in scholarly peer-reviewed journals. J Informetr. 2013;7(4):914-23. https://doi.org/10.1016/j.joi.2013.09.001

50. Johansson MA, Reich NG, Meyers LA, Lipsitch M. Preprints: an underutilized mechanism to accelerate outbreak science. PLoS Med. 2018;15(4):1-5. https://doi.org/10.1371/journal.pmed.1002549.

51. Scientific Electronic Library Online - SciELO. Preprints (pilot). São Paulo: Scientific Electronic Library Online; 2008[acesso 9 maio 2020]. Disponível em: https://preprints.scielo.org/index.php/scielo/archive

52. Ornell F, Halpern SC, Kessler FHP, Narvaez JCM. The impact of the COVID-19 pandemic on the mental health of healthcare professionals. Cad Saude Publica. 2020;36(4):1-6. https://doi.org/10.1590/0102-311×00063520

53. Ministério da Saúde (BR). Biblioteca virtual em saúde: descritores em ciências da saúde. Brasília: Ministério da Saúde; 1999 [acesso 8 maio 2020]. Disponível em: http://decs. bvs.br/cgi-bin/wxis1660.exe/decsserver/?lsisScript=../cgi-bin/ decsserver/decsserver.xis\&interface_language=p\&previous_ page=homepage\&previous_task=NULL\&task=start. Acesso em: 9 maio 2020. 2020.http://decs.bvs.br/ cgi-bin/wxis1660.exe/decsserver/?lsisScript=../cgi-bin/ decsserver/decsserver.xis\&interface_language=p\&previous_ page=homepage\&previous_task=NULL\&task=start

Contribuição dos Autores

Mota DM - Concepção, planejamento (desenho do estudo), aquisição, análise, interpretação dos dados e redação do trabalho. Ferreira PJG - Análise e redação do trabalho. Leal LF - Análise, interpretação dos dados e redação do trabalho. Todos os autores aprovaram a versão final do trabalho.

Conflito de Interesse

Os autores informam não haver qualquer potencial conflito de interesse com pares e instituições, políticos ou financeiros deste estudo. $\mathrm{Na}$ oportunidade, informamos que as opiniões, achados, conclusões e recomendações expressos neste debate são exclusivamente dos autores e não refletem a opinião oficial das suas instituições.

Esta publicação está sob a licença Creative Commons Atribuição 3.0 não Adaptada.

Para ver uma cópia desta licença, visite http://creativecommons.org/licenses/by/3.0/deed.pt_BR. 